\title{
Editorial: ACM/Springer Mobile Networks \& Applications - Special Issue on Mobile Computing and Software Engineering
}

\author{
Honghao Gao ${ }^{1} \cdot$ Yuyu Yin ${ }^{2}$ \\ Published online: 12 December 2019 \\ (C) Springer Science+Business Media, LLC, part of Springer Nature 2019
}

\section{Editorial}

With the rapid development of mobile devices, more and more individuals and enterprises move their applications into Apps and enjoy high motility services. Today we observe that Mobile Computing applications span multiple fields, such as financial payment, intelligent transportation networks, data analytics, and social networks. However, the use of mobile computing, along with this extension application, still faces challenges due to limitations, such as Requirements Analysis, Software Design, Software Testing and Verification, Software Maintain, and The Optimization Problem. All these issues have related the Principles and Practice of Software Engineering. This special issue features six selected papers with high quality.

The first article titled Dynamical Service Deployment and Replacement in Resource-Constrained Edges highlights the service deployment and replacement mechanism in MEC environment. It proposes resource-constrained service management algorithms from two perspectives. First, they design and implement a prototype of the service provisioning system that simulates the behaviors between users and servers. Second, they also propose an approach to deploy services on edge servers before the launching of these servers, and to replace services on edge servers dynamically.

The second article titled A Novel Approach to Scheduling Workflows Upon Cloud Resources with Fluctuating Performance considers that scientific computing processes to be supported by decentralized cloud infrastructures with fluctuating QoS and aim at managing the monetary cost of

Honghao Gao

gaohonghao@shu.edu.cn

Yuyu Yin

yinyuyu@hdu.edu.cn

1 Computing Center, Shanghai University, Shanghai, China

2 College of Computer, Hangzhou Dianzi University, Hangzhou, China

workflows with the completion-time constraint to be satisfied. They address the performance-variation-aware workflows scheduling problem by leveraging a time-series-based prediction model and a Critical-Path-Duration-Estimation-based (CPDE for short) VM Selection strategy.

The third article titled Long-term and Multi-step Ahead Call Traffic Forecasting with Temporal Features Mining proposes a holistic solution for forecasting long-term multi-step ahead call traffic. They give a categorized way for temporal features by studying the call traffic data. After data preprocessing, they develop an extraction method for temporal feature extraction for training the forecasting model. After that, two forecasting strategies based on taking different types of features as input are proposed.

The fourth paper titled Joint Optimization of Resource Utilization and Load Balance with Privacy Preservation for Edge Services in 5G Networks considers the mobile devices have limitations on the battery capacity, physical size, etc. an optimization problem is defined to improve the resource utilization and load balance for all the ENs while protecting the privacy information and satisfying the time requirement. Then, a balanced service offloading method, abbreviated BSOM, is proposed.

The fifth paper titled Active Defense by Mimic Association Transmission in Edge Computing considers the transmission reliability and defensive revenue reduction caused by dynamic network transformation. A real-time multi-feature anomaly detection algorithm based on Non-extensive entropy and Renyi cross entropy is proposed, and a Hidden Markov based state prediction model and a mimic transformation strategy for The Network Topology Mimic Association Graph based on predicted states is proposed. Combining these two ways improves the data transmission service quality of the active defense technology in edge computing networks.

The sixth paper titled Deep Learning Models for Real-time Human Activity Recognition with Smartphones considers the task of recognizing human activity using a smartphone's builtin accelerometer. The Human Activity Recognition (HAR) algorithm and deep learning methods are combined to reduce 
the costs of energy consumption and hardware facilities, improving the calculation speed and recognition accuracy.

Acknowledgements The guest editors are thankful to our reviewers for their effort in reviewing the manuscripts. We also thank the Edit-in-Chief, Dr. Imrich Chlamtac, for his supportive guidance during the entire process. We also thank you for the support from the National Natural Science Foundation of China (NSFC) under Grant No. 61902236.

Publisher's Note Springer Nature remains neutral with regard to jurisdictional claims in published maps and institutional affiliations.

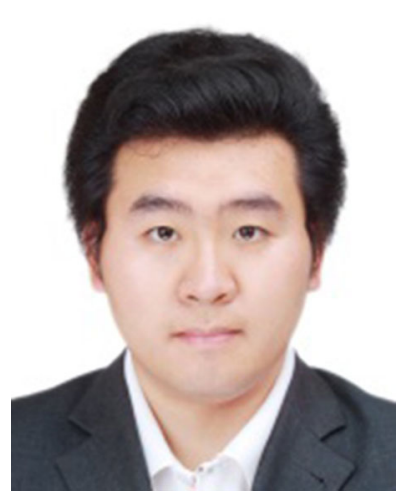

Prof. Honghao Gao, Shanghai University, China. Email: gaohonghao@shu.edu.cn Dr. Gao received the Ph.D. degree in Computer Science and started his academic career at Shanghai University in 2012. He is an IET Fellow(FIET), BCS Fellow(FBCS), EAI Fellow(FEAI), IEEE Senior Member(SMIEEE), CCF Senior Member(SMCCF), and CAAI Senior Member(SMCAAI). Dr. Gao is currently a Distinguished Professor with the Key Laboratory of Complex Systems Modeling and Simulation, Ministry of Education, China, and is a Research Fellow with the Software Engineering Information Technology Institute of Central Michigan University (CMU), USA. He is an Adjunct Professor of Gachon University, South Korea. Dr. Gao's research interests include service computing, model checking-based software verification, wireless network and IoT, and intelligent medical image processing. His researches are supported by National Natural Science Foundation of China (NSFC), Natural Science Foundation of Shanghai (NSFS), CERNET Innovation Project, and Foundation of Science and Technology Commission of Shanghai Municipality. Until now, he has approximately 40 publications in professional journals and more than 20 publications in scientific conferences including IEEE TII, IEEE TCSS, IEEE IOT, IEEE TETCI, IEEE ACCESS, FGCS, MONET, COMCOM, IJDSN, JWCN,
WCMC, IJSEKE, Autosoft, MIS, CI, FCS, SCN, AMIS, IJCM, JT, ICSW, SCC, SEKE, Mobiquitous, TASE, CollaborateCom, and SNPD, obtained 13 patent applications and registered 8 software copyrights in China involving intelligent vehicle, searching engine, cloud rendering, service composition verification and testing, and IoT application under IPv4/IPv6. He has been served as Lead Guest Editor for ACM TOMM, IEEE TITS, IEEE ACCESS, Wiley ETT, ComSIS, CI, IJDSN, MONET, WINE, JIT, JAIHC, JOEUC, JMIHI, CAI, and organized more than 20 International Conferences and Workshops, such as CollaborateCom, ChinaCom, TRIDENTCOM, Broadnest, MobiCASE, ISC, IITC, FMSC, DISA, SSIA, IEEE/ACIS ICIS and ACTI.

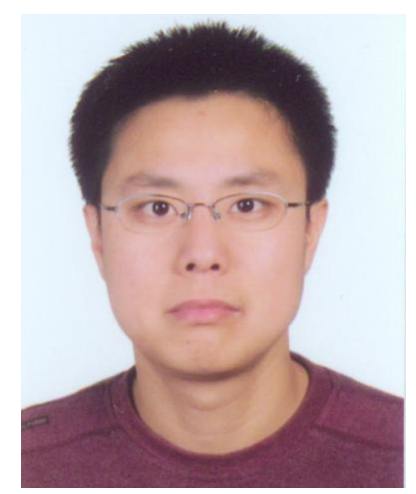

Prof. Yuyu Yin, Hangzhou Dianzi University, China. yinyuyu@hdu.edu.cn Dr. Yin received the $\mathrm{PhD}$ in Computer Science from Zhejiang University in 2010. He is currently a Professor at the College of Computer in Hangzhou Dianzi University. His research interests include Service Computing, Cloud Computing, and Business Process Management. He is an EAI Fellow, IEEE Member, Senior Member of the China Computer Federation (CCF). During the past ten years, he has published more than 50 papers in journals and refereed conferences such as MONET, IEEE ACCESS, Sensors, Entropy, IJSEKE, Mobile Information Systems, ICWS, SEKE. He organized and chaired more than 10 international conferences and workshops, such as Mobicase 2019, Collaboratecom 2018, Tridentcom 2018/2019, FMSC2011-2017, DISA201212017-2018. Also he worked as EIC of EAI Endorsed Transactions on Internet of Things, as guest editor for Journal of Information Science and Engineering and International Journal of Software Engineering and Knowledge Engineering, and as reviewers for IEEE transaction on Industry Informatics, Journal of Database Management, Future Generation Computer Systems, ect. 